International Journal of Social Science and Economic Research

ISSN: 2455-8834

Volume:05, Issue:08 "August 2020"

\title{
USE OF MAKE-UP BY YOUNG GIRLS \& WOMEN FOR IMPROVING SELF- PERCEPTION
}

\author{
${ }^{1}$ Deeksha Gumber, ${ }^{2}$ Rita Kant \\ ${ }^{1}$ M.Sc. University Institute of Fashion Technology \& Vocational Development, Panjab University, \\ Chandigarh \\ ${ }^{2}$ Faculty University Institute of Fashion Technology \& Vocational Development, Panjab University, \\ Chandigarh
}

DOI: 10.46609/IJSSER.2020.v05i08.007 URL: https://doi.org/10.46609/IJSSER.2020.v05i08.007

\begin{abstract}
Make-Up is used as a beauty aid to help build up self-esteem and confidence of an individual. This research was conducted on "Use of Make-Up by Young Girls and Women with respect to their Self- Perception" to study the preference of female respondents for various items of Make- Up and the relationship between age of female respondents with reasons and frequency of wearing Make-Up. The researcher conducted a survey for which a questionnaire was prepared and used Interview method to collect data. The sample of the study included young girls and women between the ages 15 to 55 years.

After completion of the survey, the researcher analysed the data collected. The following observations have been made during the analysis:- Younger Girls (Age group 15-25 years) in comparison to Older Women wear Make-Up to "Look Attractive" and to "Look Prettier". Among the Younger Women on the other hand, (Age group of 26-35 years) the most common reasons for wearing Make-Up are "Peer Pressure" and "Wanting to Experiment with their Looks" as well as to "Attract Peoples' Attention". In the age group of 36-45 years the reason given for wearing Make-Up was "to enhance their Overall Personality". Further among the more Senior Women (Age group 46-55 years) the reasons given for wearing Make-Up are to "Hide Flaws" and to "Hide Age". Thus the Hypothesis: Girls and Women have different reasons for wearing Make-Up is accepted. Further, the findings substantiate the results of a study conducted by Lauren A. Silverio (2009) who researched on "Makeup's effect on Self- Perception" The results of the present study also recognize that, wearing makeup does provide a certain amount of self-confidence to the wearer.
\end{abstract}

Keywords: Fashion, Makeup, Age, Woman, Young 


\section{International Journal of Social Science and Economic Research}

ISSN: $2455-8834$

Volume:05, Issue:08 "August 2020"

\section{Introduction}

Cosmetics are used to enhance ones' appearance. The importance of cosmetics has increased over the years as many people have a desire to look young and attractive. The first known people who used cosmetics to enhance their beauty were the Egyptians. With more cosmetics on the market today than ever before, it becomes obvious that they play a great role in ones' everyday life.

A research was conducted on "Use of Make-Up by Young Girls \& Women for Improving SelfPerception". The objective was to study the Reasons that led females to wear Make-Up along with Frequency of its use. To address these objectives both primary and secondary sources were explored to collect data. A survey using an Interview Schedule was used to collect data which was analysed to arrive at the results.

\section{Review of Literature}

Lauren A. Silverio (2009) studied a research on "Makeup's effect on Self-Perception" The results of the survey recognized that, with a mean score of 3.90, women do feel more put together when they wear makeup. As well with a mean score of 4.36, most women agreed to the fact that they felt good when their makeup looks good. As a result of this data it was concluded that wearing makeup does provide a certain amount of self-confidence to the wearer.

"The Effects of Self-Esteem on Makeup Involvement and Makeup Satisfaction among Elementary Students" by Hojung Lee and Heesun Oha (2017) conducted a research to analyse makeup with the intention of appearance enhancement. The purpose of the research was to elucidate how psychological traits that may affect makeup involvement and satisfaction. Results of the study, First, self-esteem had two factors, positive and negative self-esteem. Makeup involvement had two factors, pleasure pursuit and trend pursuit. Makeup satisfaction had one factor. Second, after analysing the effects of self-esteem on makeup satisfaction in makeup involvement, negative self-esteem showed positive(+) result, while positive self- esteem showed no effect. As to the effects of self-esteem on trend pursuit, positive self-esteem showed positive(+) result, while negative self-esteem showed negative(-) result. Third, after analysing the effects of self-esteem on makeup satisfaction, negative self-esteem showed positive(+) result, while positive self-esteem showed no effect.

\section{Methodology}

An instrument was developed and tested before it was finally used on a set of respondents. The sample of the study included young girls and women between the ages 15 to 55 years. The researcher used the Mall Intercept Method and identified Elante Mall, Chandigarh as the prime 


\section{International Journal of Social Science and Economic Research}

ISSN: 2455-8834

Volume:05, Issue:08 "August 2020"

location for this study. This Mall has several shops that sell international and national brand Make-Up products. The respondents chosen were visitors and buyers of cosmetic products at these shops. The sampling method being Random Sampling, every $4^{\text {th }}$ or $5^{\text {th }}$ visitor entering these shops was interviewed, covering a total of 100 respondents. Data thus collected was tabulated, coded and analysed by calculating frequencies, applying t-test to find significant differences between groups and finding correlation between variables by chi square analysis.

\section{Results and Discussion}

The questionnaire had 38 questions that enabled the researcher to study the relationship between women of different age group and their usage of makeup. They also helped the researcher understand how women feel about makeup and their usage of makeup on a daily basis. Data analysis helped to arrive at the below mentioned results.

Table 1: Reasons for use of Make-Up among young girls and women

\begin{tabular}{|l|c|c|c|c|c|c|}
\hline Reasons for use of Make-Up & Agree & $\begin{array}{c}\text { Strongly } \\
\text { Agree }\end{array}$ & Disagree & $\begin{array}{c}\text { Strongly } \\
\text { Disagree }\end{array}$ & $\begin{array}{c}\text { Can't } \\
\text { say }\end{array}$ & Total \\
\hline Look attractive & 66 & 17 & 4 & 0 & 13 & 100 \\
\hline Hide flaws & 56 & 22 & 17 & 2 & 3 & 100 \\
\hline Hide age & 27 & 11 & 43 & 14 & 5 & 100 \\
\hline Peer pressure & 11 & 14 & 46 & 20 & 9 & 100 \\
\hline Experiment with looks & 42 & 12 & 32 & 2 & 12 & 100 \\
\hline Enhance features & 68 & 19 & 4 & 0 & 9 & 100 \\
\hline Enhance over all personality & 63 & 27 & 2 & 0 & 8 & 100 \\
\hline Feel confident about yourself & 47 & 33 & 10 & 0 & 10 & 100 \\
\hline Look prettier & 61 & 28 & 2 & 0 & 9 & 100 \\
\hline To attract people's attention & 14 & 8 & 42 & 21 & 15 & 100 \\
\hline
\end{tabular}


International Journal of Social Science and Economic Research

ISSN: 2455-8834

Volume:05, Issue:08 "August 2020"

Figure 1 : Reasons for use of Make-Up among young girls and women

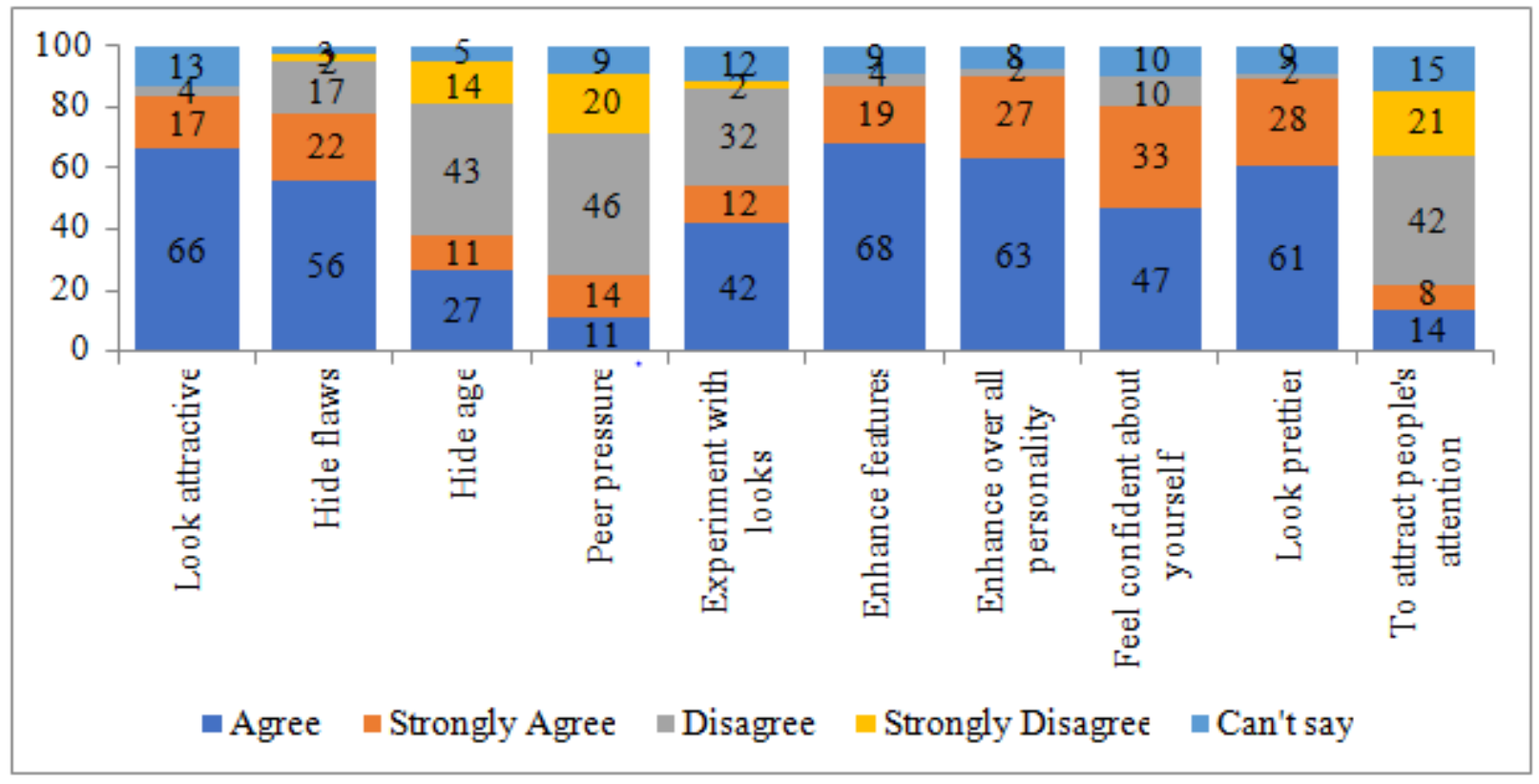

\section{Analysis of Results}

Data in Table 4.5 shows the response of persons under study with regard to reasons for wearing makeup. 68\% respondents said that make up helped enhance their features, $66 \%$ said that they wore makeup to look attractive, $63 \%$ responded that makeup enhanced their overall personality,61\% felt that they looked prettier when they wore makeup. Further $56 \%$ said that make up helped to hide flaws. While $46 \%$ respondents disagreed that they wore makeup due to peer pressure, $43 \%$ disagreed that makeup helped hide age and $42 \%$ disagreed that they wore makeup to attract peoples' attention.

Thus the main reasons to which a larger number of respondents "Agreed" to for using Make- Up were that it helped, "Enhance features" and "Look Attractive" followed by it "Enhanced overall personality" and helped "Look Prettier". Further, the largest number of respondents "Disagreed" that they used Make-Up because of "Peer Pressure" and to "Hide Age" 
International Journal of Social Science and Economic Research

ISSN: 2455-8834

Volume:05, Issue:08 "August 2020"

Table 2: Frequency of using various items of Make-Up by young girls and women

\begin{tabular}{|l|c|c|c|c|c|c|}
\hline $\begin{array}{c}\text { Frequency of using } \\
\text { Make-Up }\end{array}$ & Daily & $\begin{array}{c}\text { Few } \\
\text { times a } \\
\text { week }\end{array}$ & $\begin{array}{c}\text { Only on } \\
\text { weekends }\end{array}$ & $\begin{array}{c}\text { Occasionally } \\
\text { on special } \\
\text { events }\end{array}$ & $\begin{array}{c}\text { Parties } \\
\text { and } \\
\text { weddings }\end{array}$ & None \\
\hline Lipstick & 55 & 21 & 10 & 12 & 1 & 1 \\
\hline Eye Kohl & 18 & 23 & 6 & 28 & 4 & 21 \\
\hline Eye Liner & 10 & 16 & 8 & 34 & 18 & 14 \\
\hline BB Cream / CC Cream & 19 & 13 & 6 & 14 & 4 & 44 \\
\hline Eye Shadow & 1 & 8 & 0 & 46 & 27 & 18 \\
\hline Face Primer & 5 & 3 & 2 & 23 & 28 & 39 \\
\hline Blush & 27 & 18 & 5 & 31 & 14 & 5 \\
\hline Eyebrow Pencil & 30 & 12 & 3 & 19 & 7 & 29 \\
\hline Mascara & 24 & 19 & 6 & 33 & 9 & 9 \\
\hline Foundation & 2 & 7 & 1 & 33 & 51 & 6 \\
\hline Concealer & 9 & 11 & 1 & 32 & 27 & 20 \\
\hline Highlighter & 0 & 6 & 0 & 26 & 42 & 26 \\
\hline Contour/Bronzer & 0 & 8 & 0 & 15 & 26 & 51 \\
\hline
\end{tabular}

Figure 2: Frequency of using various items of Make-Up by young girls and women

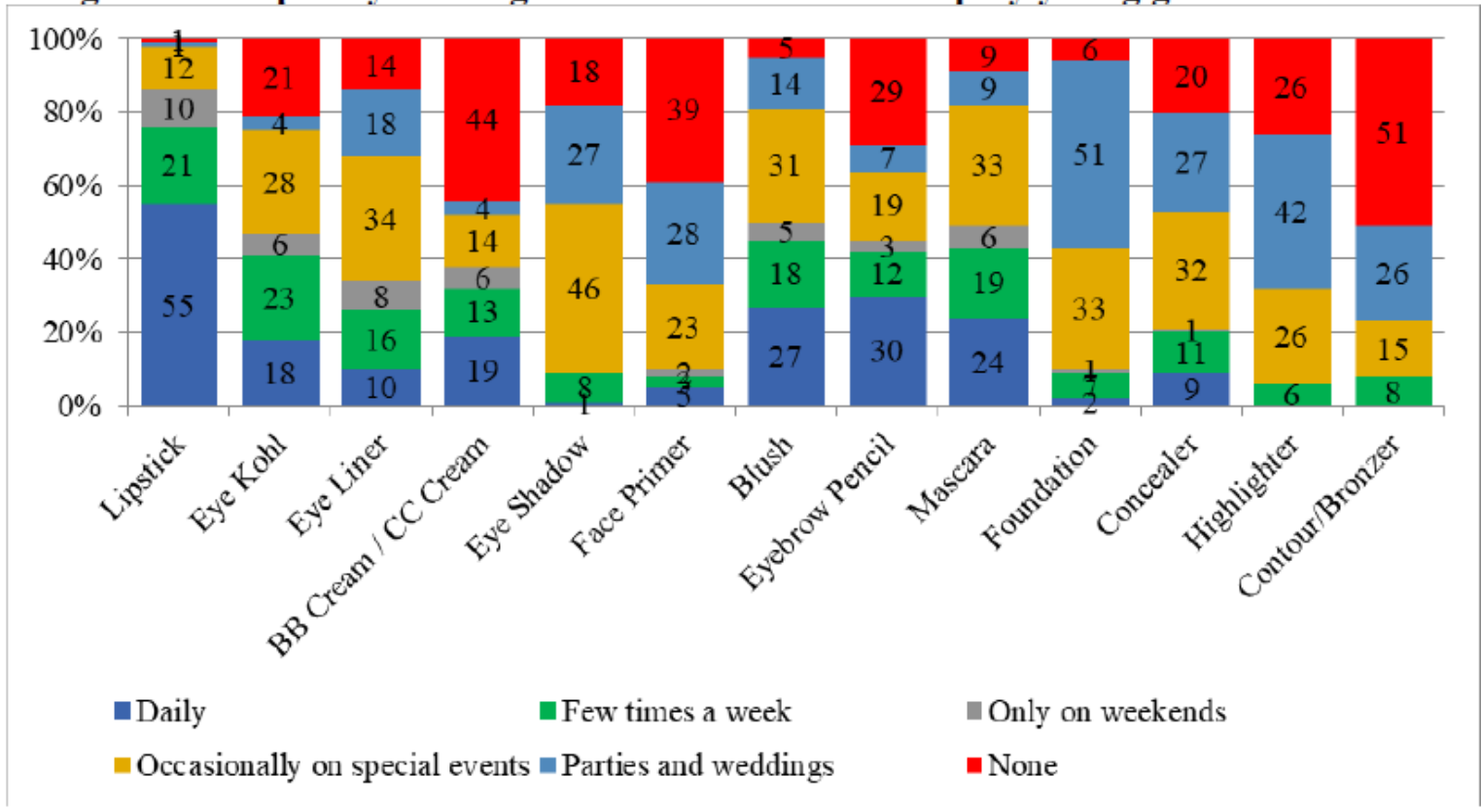

\section{Analysis of Results}

Data in Table 4.6 indicates that $55 \%$ respondents use Lipstick on a daily basis while $21 \%$ use this 


\section{International Journal of Social Science and Economic Research}

ISSN: 2455-8834

Volume:05, Issue:08 "August 2020"

item a few times a week. 23\% use Eye Kohl few times a week and 28\% use it Occasionally on Special Events.46\% use Eye Shadow Occasionally on Special Events and 34\% use Eyeliner Occasionally on Special Events.51\% use Foundation when they have to attend Parties and weddings and $42 \%$ use Highlighter when they have to attend Parties and weddings.51\% never use Contour/Bronzer and 44\% never use BB Cream/CC Cream.1\% never even use Lipstick.

Thus it can be seen that lipstick is one item that is used maximum on a daily basis by a large number of respondents followed by use of Eyebrow Pencil which is also used on a daily basis by a large number of respondents. Highlighter and Contour/Bronzer are not used on a daily basis. Eye shadow is used by a large number of respondents "Occasionally on Special events followed by Eyeliner, Mascara and Foundation. A large number of respondents never use Contour/Bronzer and BB Cream/CC Cream.

Table 3: Relationship between Age and Reasons of using Make-Up by young girls and women

\begin{tabular}{|c|c|c|c|c|c|c|c|c|c|}
\hline $\begin{array}{c}\text { Reasons } \\
\text { for } \\
\text { wearing } \\
\text { Make-Up }\end{array}$ & Responses & $\begin{array}{c}15- \\
25 \mathrm{yrs} \\
\text { n(\%) }\end{array}$ & $\begin{array}{c}\text { 26- } \\
\text { 35yrs } \\
\text { n(\%) }\end{array}$ & $\begin{array}{c}\text { 36- } \\
\mathbf{4 5 y r s} \\
\text { n(\%) }\end{array}$ & $\begin{array}{c}\text { 46- } \\
55 y r s \\
\text { n(\%) }\end{array}$ & $\begin{array}{l}\text { Total } \\
\text { n(\%) }\end{array}$ & $\begin{array}{l}\text { Chi- } \\
\text { square } \\
\text { value }\end{array}$ & df & $\begin{array}{r}\text { p- } \\
\text { value }\end{array}$ \\
\hline \multirow{4}{*}{$\begin{array}{c}\text { Look } \\
\text { attractive }\end{array}$} & Agree & \begin{tabular}{|l|}
$25(83.3)$ \\
\end{tabular} & $12(57.1)$ & $18(69.2)$ & $11(47.8)$ & $66(66)$ & \multirow{4}{*}{34.6} & \multirow{4}{*}{9} & \multirow{4}{*}{$0.00 * *$} \\
\hline & $\begin{array}{l}\text { Strongly } \\
\text { Agree }\end{array}$ & $5(16.7)$ & $9(42.9)$ & $1(3.8)$ & $2(8.7)$ & $17(17)$ & & & \\
\hline & Disagree & $0(0)$ & $0(0)$ & $2(7.7)$ & $2(8.7)$ & $4(4)$ & & & \\
\hline & Can't say & $0(0)$ & $0(0)$ & $5(19.2)$ & $8(34.8)$ & $13(13)$ & & & \\
\hline \multirow{5}{*}{ Hide flaws } & Agree & $17(56.7)$ & $8(38.1)$ & $16(61.5)$ & $15(65.2)$ & $56(56)$ & \multirow{5}{*}{20.08} & \multirow{5}{*}{12} & \multirow{5}{*}{0.07} \\
\hline & $\begin{array}{l}\text { Strongly } \\
\text { Agree }\end{array}$ & $5(16.7)$ & $10(47.6)$ & 4(15.4) & $3(13)$ & $22(22)$ & & & \\
\hline & Disagree & $5(16.7)$ & $2(9.5)$ & $5(19.2)$ & $5(21.7)$ & $17(17)$ & & & \\
\hline & $\begin{array}{l}\text { Strongly } \\
\text { Disagree }\end{array}$ & $0(0)$ & $1(4.8)$ & $1(3.8)$ & $0(0)$ & $2(2)$ & & & \\
\hline & Can't say & $3(10)$ & $0(0)$ & $0(0)$ & $0(0)$ & $3(3)$ & & & \\
\hline \multirow{6}{*}{ Hide age } & Agree & $3(10)$ & $4(19)$ & $8(30.8)$ & $12(52.2)$ & $27(27)$ & \multirow{5}{*}{26.15} & \multirow{5}{*}{12} & \multirow{5}{*}{$0.00^{* *}$} \\
\hline & $\begin{array}{l}\text { Strongly } \\
\text { Agree }\end{array}$ & $2(6.7)$ & $5(23.8)$ & $1(3.8)$ & $3(13)$ & 11(11) & & & \\
\hline & Disagree & $18(60)$ & $8(38.1)$ & 9(34.6) & $8(34.8)$ & $43(43)$ & & & \\
\hline & $\begin{array}{l}\text { Strongly } \\
\text { Disagree }\end{array}$ & 4(13.3) & $3(14.3)$ & $7(26.9)$ & $0(0)$ & $14(14)$ & & & \\
\hline & Can't say & $3(10)$ & $1(4.8)$ & $1(3.8)$ & $0(0)$ & $5(5)$ & & & \\
\hline & Agree & $3(10)$ & $3(14.3)$ & $4(15.4)$ & $1(4.3)$ & 11(11) & & & \\
\hline
\end{tabular}


International Journal of Social Science and Economic Research

ISSN: 2455-8834

Volume:05, Issue:08 "August 2020"

\begin{tabular}{|c|c|c|c|c|c|c|c|c|c|}
\hline \multirow{4}{*}{$\begin{array}{l}\text { Peer } \\
\text { pressure }\end{array}$} & $\begin{array}{c}\text { Strongly } \\
\text { Agree }\end{array}$ & $7(23.3)$ & $7(33.3)$ & $0(0)$ & $0(0)$ & $14(14)$ & \multirow{4}{*}{36.8} & \multirow{4}{*}{12} & \multirow{4}{*}{$0.00 * *$} \\
\hline & Disagree & $13(43.3)$ & $6(28.6)$ & $10(38.5)$ & 17(73.9) & $46(46)$ & & & \\
\hline & $\begin{array}{l}\text { Strongly } \\
\text { Disagree }\end{array}$ & $1(3.3)$ & $4(19)$ & $10(38.5)$ & $5(21.7)$ & $20(20)$ & & & \\
\hline & Can't say & $6(20)$ & $1(4.8)$ & $2(7.7)$ & $0(0)$ & 9(9) & & & \\
\hline \multirow{5}{*}{$\begin{array}{l}\text { Experiment } \\
\text { with looks }\end{array}$} & Agree & $17(56.7)$ & $11(52.4)$ & $10(38.5)$ & $4(17.4)$ & $42(42)$ & \multirow{4}{*}{49.1} & \multirow{4}{*}{12} & \multirow{4}{*}{$0.00 * *$} \\
\hline & $\begin{array}{l}\text { Strongly } \\
\text { Agree }\end{array}$ & $5(16.7)$ & $7(33.3)$ & $0(0)$ & $0(0)$ & $12(12)$ & & & \\
\hline & Disagree & $4(13.3)$ & $3(14.3)$ & $14(53.8)$ & $11(47.8)$ & $32(32)$ & & & \\
\hline & $\begin{array}{l}\text { Strongly } \\
\text { Disagree }\end{array}$ & $2(6.7)$ & $0(0)$ & $0(0)$ & $0(0)$ & $2(2)$ & & & \\
\hline & Can't say & $2(6.7)$ & $0(0)$ & $2(7.7)$ & $8(34.8)$ & $12(12)$ & & & \\
\hline \multirow{4}{*}{$\begin{array}{l}\text { Enhance } \\
\text { features }\end{array}$} & Agree & $21(70)$ & 10(47.6) & 19(73.1) & $18(78.3)$ & $68(68)$ & \multirow{4}{*}{27.5} & \multirow{4}{*}{9} & \multirow{4}{*}{$0.00 * *$} \\
\hline & $\begin{array}{l}\text { Strongly } \\
\text { Agree }\end{array}$ & $6(20)$ & $11(52.4)$ & $2(7.7)$ & $0(0)$ & $19(19)$ & & & \\
\hline & Disagree & $2(6.7)$ & $0(0)$ & $1(3.8)$ & $1(4.3)$ & $4(4)$ & & & \\
\hline & Can't say & $1(3.3)$ & $0(0)$ & $4(15.4)$ & $4(17.4)$ & 9(9) & & & \\
\hline \multirow{4}{*}{$\begin{array}{l}\text { Enhance } \\
\text { over all } \\
\text { personality }\end{array}$} & Agree & $17(56.7)$ & $9(42.9)$ & 20(76.9) & 17(73.9) & $63(63)$ & \multirow{4}{*}{27.1} & \multirow{4}{*}{9} & \multirow{4}{*}{$0.00^{* * *}$} \\
\hline & $\begin{array}{l}\text { Strongly } \\
\text { Agree }\end{array}$ & 11(36.7) & $12(57.1)$ & $3(11.5)$ & $1(4.3)$ & $27(27)$ & & & \\
\hline & Disagree & $1(3.3)$ & $0(0)$ & $1(3.8)$ & $0(0)$ & $2(2)$ & & & \\
\hline & Can't say & $1(3.3)$ & $0(0)$ & $2(7.7)$ & $5(21.7)$ & $8(8)$ & & & \\
\hline \multirow{4}{*}{$\begin{array}{l}\text { Feel } \\
\text { confident } \\
\text { about } \\
\text { yourself }\end{array}$} & Agree & 14(46.7) & $10(47.6)$ & $12(46.2)$ & $11(47.8)$ & $47(47)$ & \multirow{4}{*}{11.2} & \multirow{4}{*}{9} & \multirow{4}{*}{0.26} \\
\hline & $\begin{array}{l}\text { Strongly } \\
\text { Agree }\end{array}$ & $9(30)$ & 10(47.6) & $6(23.1)$ & $8(34.8)$ & $33(33)$ & & & \\
\hline & Disagree & $5(16.7)$ & $1(4.8)$ & $2(7.7)$ & $2(8.7)$ & $10(10)$ & & & \\
\hline & Can't say & $2(6.7)$ & $0(0)$ & $6(23.1)$ & $2(8.7)$ & $10(10)$ & & & \\
\hline \multirow{4}{*}{$\begin{array}{l}\text { Look } \\
\text { prettier }\end{array}$} & Agree & $22(73.3)$ & $11(52.4)$ & $15(57.7)$ & $13(56.5)$ & $61(61)$ & \multirow{4}{*}{22.6} & \multirow{4}{*}{9} & \multirow{4}{*}{$0.00 * *$} \\
\hline & $\begin{array}{l}\text { Strongly } \\
\text { Agree }\end{array}$ & $8(26.7)$ & $10(47.6)$ & $3(11.5)$ & 7(30.4) & $28(28)$ & & & \\
\hline & Disagree & $0(0)$ & $0(0)$ & $1(3.8)$ & $1(4.3)$ & $2(2)$ & & & \\
\hline & Can't say & $0(0)$ & $0(0)$ & $7(26.9)$ & $2(8.7)$ & $9(9)$ & & & \\
\hline \multirow{5}{*}{$\begin{array}{l}\text { To attract } \\
\text { people's } \\
\text { attention }\end{array}$} & Agree & $7(23.3)$ & $3(14.3)$ & $4(15.4)$ & $0(0)$ & $14(14)$ & \multirow{5}{*}{35.7} & \multirow{5}{*}{12} & \multirow{5}{*}{$0.00 * *$} \\
\hline & $\begin{array}{l}\text { Strongly } \\
\text { Agree }\end{array}$ & $3(10)$ & $5(23.8)$ & $0(0)$ & $0(0)$ & $8(8)$ & & & \\
\hline & Disagree & $10(33.3)$ & $7(33.3)$ & $13(50)$ & $12(52.2)$ & $42(42)$ & & & \\
\hline & $\begin{array}{l}\text { Strongly } \\
\text { Disagree }\end{array}$ & $1(3.3)$ & $3(14.3)$ & $7(26.9)$ & $10(43.5)$ & $21(21)$ & & & \\
\hline & Can't say & $9(30)$ & $3(14.3)$ & $2(7.7)$ & $1(4.3)$ & $15(15)$ & & & \\
\hline
\end{tabular}

$* *$ Significant at 0.01 level 
International Journal of Social Science and Economic Research

ISSN: 2455-8834

Volume:05, Issue:08 "August 2020"

\section{Analysis of Results}

Table 4.17 shows a significant relationship between age of the respondents and various reasons why they wear Make-Up (Looking Attractive, Hiding Age, Peer Pressure, and Experimenting with Looks, Enhancing Features, Enhancing overall Personality, Looking Prettier and Attracting Peoples' Attention are reasons that have significant relationship with Age) at $>0.01$ Level of significance. Among the 15-25 years age group $83.3 \%$ agree and $16.7 \%$ strongly agree that they wear Make-Up to Look Attractive. In this same age group, $73.3 \%$ and $26.7 \%$ agree and strongly agree that they wear Make-Up to Look Prettier. Among the 46-55 years age group 65.2\% agree and $13 \%$ strongly agree that wear Make-Up to Hide Flaws. Further in this same age group 52.2\% agree and $13 \%$ strongly agree that they wear Make-Up to Hide Age.In the age group of 26-35 years $14.3 \%$ and $33.3 \%$ agree and strongly agree that they wear Make-Up due to Peer Pressure and $52.4 \%$ and $33.3 \%$ agree and strongly agree that they wear Make-Up to Experiment with their Looks. $14.3 \%$ and $23.8 \%$ respondents in this age group also said that they wear Make-Up to Attract Peoples' attention. In the age group of 36- 45 years $76.9 \%$ and $11.5 \%$ respondents said that they wear makeup to Enhance their Overall Personality.

Thus it can be concluded that the younger girls (Age group 15-25 years) in comparison to older women wear Make-Up to "Look Attractive" and to "Look Prettier". Among the younger women on the other hand, (age group of 26-35 years) the most common reasons for wearing Make-Up are "Peer Pressure" and "Wanting to Experiment with their Looks" as well as to "Attract Peoples' attention".In the age group of 36-45 years the reason given for wearing Make- Up was "to Enhance their Overall Personality". Further among the more senior women (Age group 46-55 years) the reasons given for wearing Make-Up are to "Hide Flaws" and to "Hide Age"

Table 4: Relationship between Age of respondents and Frequency of using Make-Up

\begin{tabular}{|c|c|c|c|c|c|c|c|c|c|}
\hline $\begin{array}{l}\text { Makeup } \\
\text { products }\end{array}$ & $\begin{array}{l}\text { Practice of } \\
\text { using } \\
\text { makeup }\end{array}$ & $\begin{array}{c}15- \\
25 y r s \\
n(\%)\end{array}$ & $\begin{array}{c}\text { 26- } \\
\text { 35yrs } \\
\text { n(\%) }\end{array}$ & $\begin{array}{l}\text { 36- } \\
45 y r s \\
\text { n(\%) }\end{array}$ & $\begin{array}{c}\text { 46- } \\
\text { 55yrs } \\
\text { n(\%) }\end{array}$ & $\begin{array}{l}\text { Total } \\
\text { n(\%) }\end{array}$ & $\begin{array}{l}\text { Chi- } \\
\text { square } \\
\text { value }\end{array}$ & Df & $\begin{array}{c}\text { p- } \\
\text { value }\end{array}$ \\
\hline \multirow{5}{*}{ Lipstick } & Daily & 19(63.3) & $15(71.4)$ & $17(65.4)$ & $4(17.4)$ & $55(55)$ & \multirow{5}{*}{32.6} & \multirow{5}{*}{15} & \multirow{5}{*}{$0.00^{* *}$} \\
\hline & $\begin{array}{l}\text { Few times a } \\
\text { week }\end{array}$ & $7(23.3)$ & $3(14.3)$ & $5(19.2)$ & $6(26.1)$ & $21(21)$ & & & \\
\hline & \begin{tabular}{|l|} 
Only on \\
weekends
\end{tabular} & $0(0)$ & $1(4.8)$ & $2(7.7)$ & $7(30.4)$ & $10(10)$ & & & \\
\hline & $\begin{array}{l}\text { Occasionally } \\
\text { on special } \\
\text { events }\end{array}$ & $3(10)$ & $2(9.5)$ & $1(3.8)$ & $6(26.1)$ & $12(12)$ & & & \\
\hline & $\begin{array}{l}\text { Parties and } \\
\text { weddings }\end{array}$ & $1(3.3)$ & $0(0)$ & $0(0)$ & $0(0)$ & 1(1) & & & \\
\hline
\end{tabular}


International Journal of Social Science and Economic Research

ISSN: $2455-8834$

Volume:05, Issue:08 "August 2020"

\begin{tabular}{|c|c|c|c|c|c|c|c|c|c|}
\hline & Never & $0(0)$ & $0(0)$ & $1(3.8)$ & $0(0)$ & $1(1)$ & & & \\
\hline \multirow{6}{*}{ Eye kohl } & Daily & $5(16.7)$ & $6(28.6)$ & $7(26.9)$ & $0(0)$ & $18(18)$ & \multirow{6}{*}{36.5} & \multirow{6}{*}{15} & \multirow{6}{*}{$0.00 * *$} \\
\hline & $\begin{array}{l}\text { Few times a } \\
\text { week }\end{array}$ & $9(30)$ & $5(23.8)$ & $6(23.1)$ & $3(13)$ & $23(23)$ & & & \\
\hline & \begin{tabular}{|l|} 
Only on \\
weekends
\end{tabular} & $0(0)$ & $1(4.8)$ & 1(3.8) & $4(17.4)$ & $6(6)$ & & & \\
\hline & $\begin{array}{l}\text { Occasionally } \\
\text { on special } \\
\text { events }\end{array}$ & $15(50)$ & $5(23.8)$ & $2(7.7)$ & $6(26.1)$ & $28(28)$ & & & \\
\hline & $\begin{array}{l}\text { Parties and } \\
\text { weddings }\end{array}$ & $0(0)$ & $1(4.8)$ & $1(3.8)$ & $2(8.7)$ & 4(4) & & & \\
\hline & Never & $1(3.3)$ & $3(14.3)$ & $9(34.6)$ & $8(34.8)$ & $21(21)$ & & & \\
\hline \multirow{6}{*}{ Eye liner } & Daily & $2(6.7)$ & $3(14.3)$ & $5(19.2)$ & $0(0)$ & $10(10)$ & \multirow{6}{*}{39.4} & \multirow{6}{*}{15} & \multirow{6}{*}{$0.00 * *$} \\
\hline & $\begin{array}{l}\text { Few times a } \\
\text { week }\end{array}$ & $8(26.7)$ & $7(33.3)$ & $1(3.8)$ & $0(0)$ & $16(16)$ & & & \\
\hline & \begin{tabular}{|l|} 
Only on \\
weekends
\end{tabular} & $2(6.7)$ & $2(9.5)$ & $0(0)$ & $4(17.4)$ & $8(8)$ & & & \\
\hline & $\begin{array}{l}\text { Occasionally } \\
\text { on special } \\
\text { events }\end{array}$ & $13(43.3)$ & $5(23.8)$ & $12(46.2)$ & $4(17.4)$ & $34(34)$ & & & \\
\hline & $\begin{array}{l}\text { Parties and } \\
\text { weddings }\end{array}$ & $3(10)$ & $3(14.3)$ & $3(11.5)$ & $9(39.1)$ & $18(18)$ & & & \\
\hline & Never & $2(6.7)$ & $1(4.8)$ & $5(19.2)$ & $6(26.1)$ & $14(14)$ & & & \\
\hline \multirow{6}{*}{$\begin{array}{l}\text { BB Cream } \\
\text { / CC } \\
\text { Cream }\end{array}$} & Daily & $9(30)$ & $6(28.6)$ & $4(15.4)$ & $0(0)$ & 19(19) & \multirow{6}{*}{49.5} & \multirow{6}{*}{15} & \multirow{6}{*}{$0.00 * *$} \\
\hline & $\begin{array}{l}\text { Few times a } \\
\text { week }\end{array}$ & $10(33.3)$ & $1(4.8)$ & $2(7.7)$ & $0(0)$ & $13(13)$ & & & \\
\hline & \begin{tabular}{|l|}
$\begin{array}{l}\text { Only on } \\
\text { weekends }\end{array}$ \\
\end{tabular} & $2(6.7)$ & $1(4.8)$ & 2(7.7) & $1(4.3)$ & $6(6)$ & & & \\
\hline & $\begin{array}{l}\text { Occasionally } \\
\text { on special } \\
\text { events }\end{array}$ & $4(13.3)$ & $5(23.8)$ & $4(15.4)$ & $1(4.3)$ & $14(14)$ & & & \\
\hline & $\begin{array}{l}\text { Parties and } \\
\text { weddings }\end{array}$ & $3(10)$ & $0(0)$ & $0(0)$ & $1(4.3)$ & 4(4) & & & \\
\hline & Never & $2(6.7)$ & $8(38.1)$ & $14(53.8)$ & $20(87)$ & $44(44)$ & & & \\
\hline \multirow{4}{*}{$\begin{array}{l}\text { Eye } \\
\text { shadow }\end{array}$} & Daily & $1(3.3)$ & $0(0)$ & $0(0)$ & $0(0)$ & $1(1)$ & \multirow{4}{*}{45.2} & \multirow{4}{*}{12} & \multirow{4}{*}{$0.00 * *$} \\
\hline & $\begin{array}{l}\text { Few times a } \\
\text { week }\end{array}$ & $1(3.3)$ & $6(28.6)$ & $1(3.8)$ & $0(0)$ & $8(8)$ & & & \\
\hline & $\begin{array}{l}\text { Occasionally } \\
\text { on special } \\
\text { events }\end{array}$ & $17(56.7)$ & $10(47.6)$ & $16(61.5)$ & $3(13)$ & $46(46)$ & & & \\
\hline & $\begin{array}{l}\text { Parties and } \\
\text { weddings }\end{array}$ & $9(30)$ & $4(19)$ & $6(23.1)$ & $8(34.8)$ & $27(27)$ & & & \\
\hline
\end{tabular}


International Journal of Social Science and Economic Research

ISSN: 2455-8834

Volume:05, Issue:08 "August 2020"

\begin{tabular}{|c|c|c|c|c|c|c|c|c|c|}
\hline & Never & $2(6.7)$ & $1(4.8)$ & $3(11.5)$ & $12(52.2)$ & $18(18)$ & & & \\
\hline \multirow{6}{*}{$\begin{array}{l}\text { Face } \\
\text { Primer }\end{array}$} & Daily & $0(0)$ & $5(23.8)$ & $0(0)$ & $0(0)$ & $5(5)$ & \multirow{6}{*}{58.9} & \multirow{6}{*}{15} & \multirow{6}{*}{$0.00^{* *}$} \\
\hline & $\begin{array}{l}\text { Few times a } \\
\text { week }\end{array}$ & $1(3.3)$ & $2(9.5)$ & $0(0)$ & $0(0)$ & $3(3)$ & & & \\
\hline & \begin{tabular}{|l|} 
Only on \\
weekends
\end{tabular} & $1(3.3)$ & $1(4.8)$ & $0(0)$ & $0(0)$ & $2(2)$ & & & \\
\hline & $\begin{array}{l}\text { Occasionally } \\
\text { on special } \\
\text { events }\end{array}$ & $14(46.7)$ & $5(23.8)$ & $4(15.4)$ & $0(0)$ & $23(23)$ & & & \\
\hline & $\begin{array}{l}\text { Parties and } \\
\text { weddings }\end{array}$ & $8(26.7)$ & $5(23.8)$ & $11(42.3)$ & $4(17.4)$ & $28(28)$ & & & \\
\hline & Never & $6(20)$ & $3(14.3)$ & 11(42.3) & 19(82.6) & $39(39)$ & & & \\
\hline \multirow{6}{*}{ Blush } & Daily & $7(23.3)$ & $10(47.6)$ & $9(34.6)$ & $1(4.3)$ & $27(27)$ & \multirow{3}{*}{31.4} & \multirow{3}{*}{15} & \multirow{3}{*}{$0.01 * *$} \\
\hline & $\begin{array}{l}\text { Few times a } \\
\text { week }\end{array}$ & $6(20)$ & $5(23.8)$ & $6(23.1)$ & $1(4.3)$ & $18(18)$ & & & \\
\hline & \begin{tabular}{|l|} 
Only on \\
weekends
\end{tabular} & $1(3.3)$ & $0(0)$ & $3(11.5)$ & $1(4.3)$ & $5(5)$ & & & \\
\hline & $\begin{array}{l}\text { Occasionally } \\
\text { on special } \\
\text { events }\end{array}$ & $10(33.3)$ & $5(23.8)$ & $5(19.2)$ & $11(47.8)$ & $31(31)$ & & & \\
\hline & $\begin{array}{l}\text { Parties and } \\
\text { weddings }\end{array}$ & $5(16.7)$ & $0(0)$ & $1(3.8)$ & $8(34.8)$ & $14(14)$ & & & \\
\hline & Never & $1(3.3)$ & $1(4.8)$ & $2(7.7)$ & $1(4.3)$ & $5(5)$ & & & \\
\hline \multirow{6}{*}{$\begin{array}{l}\text { Eyebrow } \\
\text { Pencil }\end{array}$} & Daily & $10(33.3)$ & $11(52.4)$ & $7(26.9)$ & $2(8.7)$ & $30(30)$ & \multirow{6}{*}{20.8} & \multirow{6}{*}{15} & \multirow{6}{*}{0.14} \\
\hline & $\begin{array}{l}\text { Few times a } \\
\text { week }\end{array}$ & $3(10)$ & $3(14.3)$ & $3(11.5)$ & $3(13)$ & $12(12)$ & & & \\
\hline & \begin{tabular}{|l|} 
Only on \\
weekends
\end{tabular} & $0(0)$ & $0(0)$ & $2(7.7)$ & $1(4.3)$ & $3(3)$ & & & \\
\hline & $\begin{array}{l}\text { Occasionally } \\
\text { on special } \\
\text { events }\end{array}$ & $6(20)$ & $4(19)$ & $5(19.2)$ & $4(17.4)$ & 19(19) & & & \\
\hline & $\begin{array}{l}\text { Parties and } \\
\text { weddings }\end{array}$ & $4(13.3)$ & $1(4.8)$ & $1(3.8)$ & $1(4.3)$ & $7(7)$ & & & \\
\hline & Never & $7(23.3)$ & $2(9.5)$ & $8(30.8)$ & $12(52.2)$ & $29(29)$ & & & \\
\hline \multirow[b]{4}{*}{ Mascara } & Daily & $8(26.7)$ & $3(14.3)$ & $6(23.1)$ & $7(30.4)$ & $24(24)$ & \multirow[b]{4}{*}{19.1} & \multirow[b]{4}{*}{15} & \multirow[b]{4}{*}{0.21} \\
\hline & $\begin{array}{l}\text { Few times a } \\
\text { week }\end{array}$ & $9(30)$ & $3(14.3)$ & $4(15.4)$ & $3(13)$ & 19(19) & & & \\
\hline & \begin{tabular}{|l|} 
Only on \\
weekends
\end{tabular} & $1(3.3)$ & $0(0)$ & $3(11.5)$ & $2(8.7)$ & $6(6)$ & & & \\
\hline & $\begin{array}{l}\text { Occasionally } \\
\text { on special } \\
\text { events }\end{array}$ & $7(23.3)$ & $12(57.1)$ & $9(34.6)$ & $5(21.7)$ & $33(33)$ & & & \\
\hline
\end{tabular}


International Journal of Social Science and Economic Research

ISSN: 2455-8834

Volume:05, Issue:08 "August 2020"

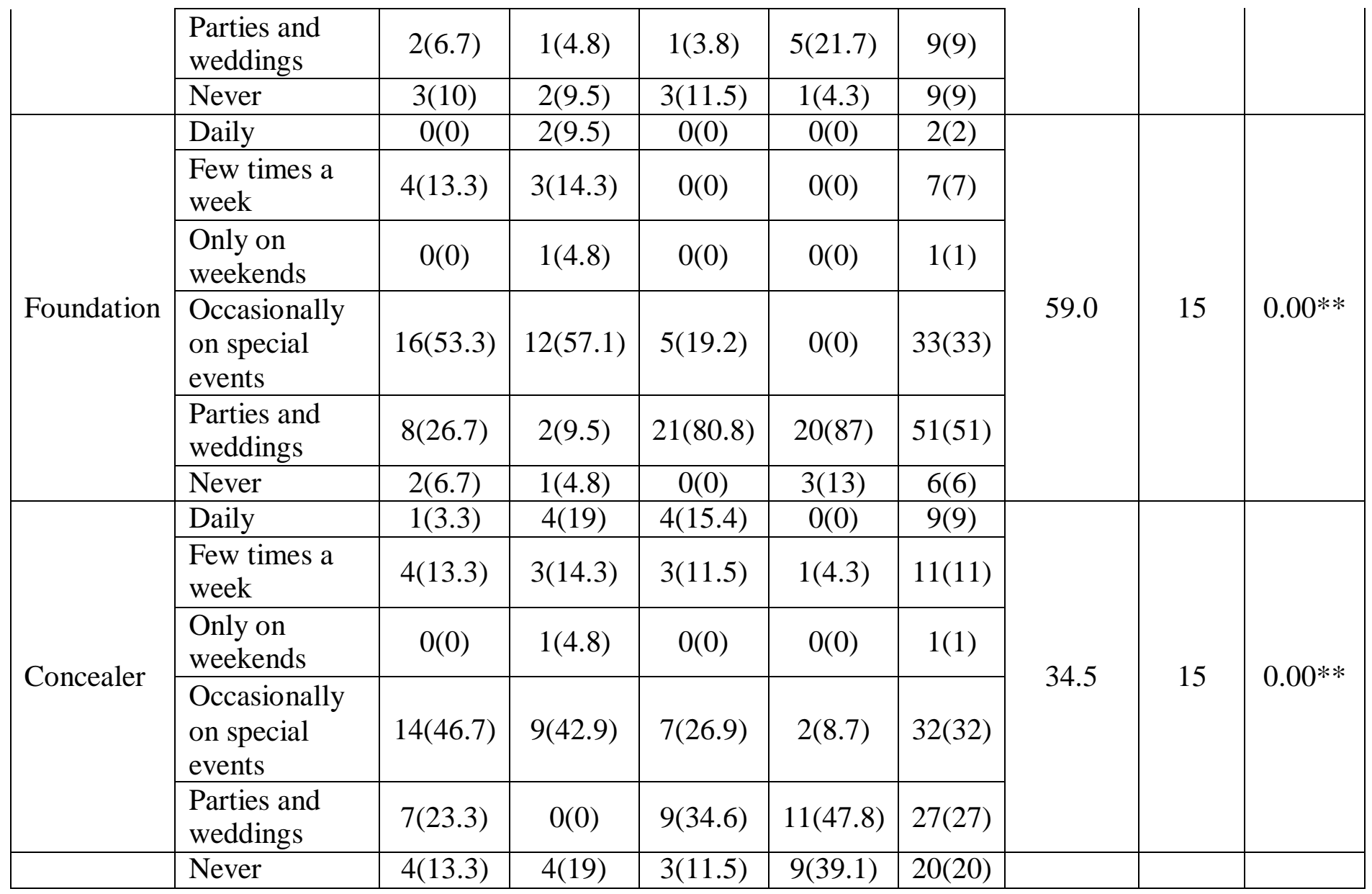

\section{Analysis of Results}

Table 4.18 shows a significant relationship between age of the respondents and the frequency of using different makeup products. (Lipstick, Eye Kohl, Eye Liner, BB Cream/CC Cream, Eye Shadow, Face Primer, Blush, Eyebrow Pencil, Mascara, Foundation, Concealer) at $>0.01$ Level of significance.

Among the age group 15-25 years, 63\% wear lipstick, $16.7 \%$ wear eye kohl, $6.7 \%$ wear eye liner, $30 \%$ wear BB cream/ CC cream, $3.3 \%$ wear eye shadow and concealer, $23.3 \%$ wear blush, $33.3 \%$ wear eyebrow pencil, $26.7 \%$ wear mascara on daily basis. Among the age group 26-35 years, $71.4 \%$ wear lipstick, $28.6 \%$ wear eye kohl and BB cream/ CC cream, $14.3 \%$ wear eye liner, $23.8 \%$ wear face primer, $47.6 \%$ wear blush, $52.4 \%$ wear eyebrow pencil, $14.3 \%$ wear mascara, 9.5\% wear foundation, $19 \%$ wear concealer on daily basis. Among the age group 36- 45 years, $65.4 \%$ wear lipstick, $26.9 \%$ wear eye kohl, $15 \%$ wear BB cream/ CC cream, $26.9 \%$ wear eye liner, $34.6 \%$ wear blush, $26.9 \%$ wear eyebrow pencil, $23.1 \%$ wear mascara, $15.4 \%$ wear concealer on daily basis whereas no respondents wear eye shadow, foundation and face primer 


\section{International Journal of Social Science and Economic Research}

ISSN: $2455-8834$

Volume:05, Issue:08 "August 2020"

on daily basis. Among the age group 46-55 years, $17.4 \%$ wear lipstick, $4.3 \%$ wear blush, $8.7 \%$ wear eyebrow pencil, 30.4\% wear mascara on daily basis whereas no respondents wear eye kohl, eye liner, BB cream/ CC cream, eye shadow, face primer, foundation, concealer on daily basis. Use of Make-Up products on a daily basis reduces in 45-55 age group respondents in comparison to the 15-25 age group respondents; more so in case of Lipstick, Eye Kohl, BB Cream/CC Cream and Blush. In fact larger number of respondents in the age group of 15-25 Years showed the use of All the Make-Up products. On the other hand larger number of respondents in the Age group of 45-55 years showed a reduced use of Make-Up items on a Daily basis. Further on a daily basis, respondents in the age group 25-35 years and 35-45 years showed lesser use of Make-Up items in comparison to the 15-25 years age group but more use of these items as compared to the use of Make-Up items by 45-55 years age group respondents. This trend is also seen in wearing Make-Up "Occasionally and on Special Events" where a larger number of Youngest Age Group Respondents are seen to use Make-Up items as compared to the older Age group respondents.

\section{Conclusion}

Based on analysis and interpretation of data it was concluded that a larger number of respondents agreed that use of Make-Up was increasing among young girls.It was seen that $68 \%$ respondents said that Make-Up helped enhance their features.66\% said that they wore Make-Up to look attractive. $63 \%$ responded that Make-Up enhanced their overall personality. $61 \%$ felt that they looked prettier when they wore Make-Up. 56\% said that Make- Up helped to hide flaws.46\% respondents disagreed that they wore Make-Up due to peer pressure.43\% disagreed that MakeUp helped hide age. $42 \%$ disagreed that they wore Make- Up to attract peoples' attention.

Further it was observed that Younger Girls (Age group 15-25 years) in comparison to Older Women wear Make-Up to "Look Attractive" and to "Look Prettier". Among the Younger Women on the other hand, (Age group of 26-35 years) the most common reasons for wearing Make-Up are "Peer Pressure" and "Wanting to Experiment with their Looks" as well as to “Attract Peoples' Attention”. In the age group of 36-45 years the reason given for wearing MakeUp was "to Enhance their Overall Personality". Further among the more Senior Women (Age group 46-55 years) the reasons given for wearing Make-Up are to "Hide Flaws" and to "Hide Age". The findings substantiate the results of a study conducted by Lauren A. Silverio (2009) who researched on "Makeup's effect on Self-Perception" The results of the survey recognized that, wearing makeup does provide a certain amount of self-confidence to the wearer.

\section{References}

1. Ya-Ning Lee (2008) "Make-Up Time: A Study Of Morning Grooming Time In The U.S." 46 
International Journal of Social Science and Economic Research

ISSN: 2455-8834

Volume:05, Issue:08 "August 2020"

pages https://etda.libraries.psu.edu/files/final submissions/6776 17 July,2020

2. Dr. B. Suresh Lal, N.V. Sreeranga Prasad \& G. Kavitha (2008) "Spending Pattern on Cosmetic Products in Rural Areas: A Case Study" https://www.researchgate.net/publication/338832352_Spending_Pattern_on_Cosmetic _Products_in_Rural_Areas_A_Case_Study 17 July,2020

3. Lauren A. Silverio (2009) "Makeup's effect on Self-Perception" 48 pages. https://pdfs.semanticscholar.org/a881/ac5b88bb85acb1b50cf7ffd4dbf25fd3850f.pdf? $\mathrm{ga}=2.18591153 .926319659 .1595008467-1143103072.159500846717$ July,2020

4. Ann Marie Britton (2012) “The Beauty Industry's Influence on Women in Society" 41 pages https://scholars.unh.edu/cgi/viewcontent.cgi?article=1085\&context=honors 17 July,2020

5. Hojung Lee and Heesun Oh (2017) "The Effects of Self-Esteem on Makeup Involvement and Makeup Satisfaction among Elementary Students" 9 pages. Archives of design research 2018. 05. vol 31. no 2. http://www.aodr.org/xml/13800/13800.pdf 17 July,2020

6. Anjana S S (2018) "A Study On Factors Influencing Cosmetic Buying Behaviour Of Consumers" http://iaraedu.com/pdf/ijair-volume-6-issue-2-vii-april\%E2\%80\%93june- 2019part-2.pdf 17 July,2020 